\title{
HEAT TRANSFER STUDY IN SLUG FLOW ON ELLIPTICAL DUCTS CROSS SECTIONS BY GENERALIZED INTEGRAL TRANSFORM TECHNIQUE
}

\author{
C. R. M. Maiaa, \\ R. A. V. Ramos, \\ M. F. Pelegrini, \\ and T. A. Alves \\ Universidade Estadual Paulista - UNESP \\ Departamento de Engenharia Mecânica \\ Av. Brasil, 56, Centro \\ 15385-000, Ilha Solteira, SP, Brasil \\ acassio@dem.feis.unesp.br

\begin{abstract}
This work shows the calculation of heat transfer parameters for slug flow in the thermal entrance region of elliptical section tubes submitted to a second kind boundary condition. The main difficulty in the application of the boundary conditions in problems with this kind of geometry has been removed by using a suitable coordinate change. The generalized integral transform technique (GITT) has been used to obtain the solution of the energy equation. The mixture temperature and the local and average Nusselt numbers have been calculated for several aspect ratios and the results have been compared with those found in the literature.

Keywords: Forced convection, slug flow, integral transform, mixture temperature, elliptical ducts.
\end{abstract}

\section{NOMENCLATURE}

a major ellipsis semi-axis, $\mathrm{m}$

$A_{i j} \quad$ non transformed coefficient

$A_{s c}$

$b$

$B_{i j m n}$

$c_{p}$

$C_{i}$

$D_{i m}$

$D_{h}$

G

$h$

$h_{u}$

$h_{v}$

H

$J$

$k$

$k$ fluid conductivity, W/m.K

$K_{i} \quad$ normalized eigenfunction

$L_{\text {th }} \quad$ thermal entry length

$M_{m} \quad$ normalization integral

$N_{i} \quad$ normalization integral

$\mathrm{Nu} \quad$ local Nusselt number

$N u_{a v} \quad$ average Nusselt number

$P \quad$ perimeter of the elliptical contour, $\mathrm{m}$

$P e \quad$ Peclet number

Pr Prandtl number

$\dot{q}_{0}^{\prime \prime} \quad$ heat flux at the contour, $\mathrm{W} / \mathrm{m}^{2}$

Re Reynolds number

$T$ temperature, $\mathrm{K}$

$T_{a v} \quad$ average temperature, $\mathrm{K}$

$T_{w, a v}^{a v} \quad$ average wall temperature, $\mathrm{K}$
$T_{0} \quad$ constant inlet temperature, $\mathrm{K}$

$u, v \quad$ dimensionless elliptical coordinates

$u_{a v} \quad$ specific internal energy, $\mathrm{kJ} / \mathrm{kg}$

$v_{0} \quad$ coordinate contour

$w \quad$ velocity field, $\mathrm{m} / \mathrm{s}$

$w_{0} \quad$ velocity profile for fully developed laminar flow, $\mathrm{m} / \mathrm{s}$

$x, y, z \quad$ coordinate axis, $\mathrm{m}$

$X, Y, Z$ dimensionless coordinate

$Z_{m} \quad$ normalized eigenfunction

\section{Greek symbols}

$\alpha \quad$ dimensionless ellipsis semi-axis

$\alpha^{*} \quad$ dimensionless focal distance

$\beta \quad$ dimensionless ellipsis semi-axis

$\Gamma \quad$ cross section contour

$\varphi_{m} \quad$ eigenfunction related to the coordinate $\mathrm{v}$

$\eta \quad$ normal coordinate, $\mathrm{m}$

$\eta^{*} \quad$ dimensionless normal coordinate

$\kappa \quad$ ellipsis semi-axis ratio

$\lambda_{m} \quad$ eigenvalue associated to the $\phi_{\mathrm{m}}(\mathrm{v})$

$\mu_{i} \quad$ eigenvalue associated to the $\varnothing_{\mathrm{i}}(\mathrm{u})$

$\theta$ dimensionless temperature

$\theta_{a v}$ dimensionless average temperature

$\theta_{w, a v}$ dimensionless average wall temperature

$\theta^{*}$ dimensionless temperature

$\widetilde{\theta}^{*} \quad$ transformed potential in u direction

$\widetilde{\widetilde{\theta}}^{*} \quad$ transformed potential in v direction

$\rho \quad$ fluid density, $\mathrm{kg} / \mathrm{m}^{3}$

$\rho_{\text {asp }} \quad$ aspect ratio

$\Omega \quad$ cross section domain

$\psi_{i} \quad$ eigenfunction related to the coordinate $\mathrm{u}$ 


\section{Subscripts}

$i \quad$ integer index

$j \quad$ integer index

$m \quad$ integer index

$n \quad$ integer index

\section{INTRODUCTION}

Although many research works have been dedicated to forced convection problems in ducts, analytical solutions for complex mass and heat transfer problems are not commonly found in the literature (Shah and London, 1978; Kakaç et al., 1998).

The major drawback to derive analytical solutions for that class of problems resides in the impossibility of employing the method of separation of variables. With the development of the Generalized Integral Transform Technique (GITT) this obstacle has been somewhat overcome, making it possible to obtain solutions for the most varied and complex diffusion problems (Aparecido, 1997). Problems such as diffusion ducts with irregular shapes (Maia et al., 2000), time varying coefficients and boundary conditions (Cotta and Özisik, 1986; Cotta and Özisik, 1987), space dependence for the heat transfer coefficients and boundary conditions (Vick and Wells, 1986), thermally and hydrodynamically developing flows (Alves et al., 2004; Pelegrini et al., 2004 and Silva and Cotta, 1992), diffusion involving moving boundaries (Diniz et al., 1990), and non Newtonian fluids (Maia et al., 2004) have all been provided a suitable analytical solution, albeit not always in a closed expression.

In the present work, heat transfer parameters calculation for the laminar thermally developing flow problem in ducts of elliptical section with boundary condition of second kind, a setup itself not amenable to variable separation, has been presented. Regarding the boundary conditions, an additional difficulty is due to the non-regular two dimensional characteristic of the cross section of the elliptical duct. A suitable change of variables was devised to transform the elliptic profile into a new geometry, simplifying the application of the boundary conditions. The Generalized Integral Transform Technique was later applied to the energy equation to obtain the temperature field in the flow and, consequently, to determine the parameters of interest.

\section{ANALYSIS}

In the formulation of the present problem, thermally and hydrodynamically developing laminar steady state flow has been assumed. The effects of viscous dissipation and axial conduction have been neglected and constant fluid properties have been admitted constant throughout. Therefore, the energy equation becomes:

$$
\rho c_{p} w(x, y) \frac{\partial T}{\partial z}=k\left[\frac{\partial^{2} T}{\partial x^{2}}+\frac{\partial^{2} T}{\partial y^{2}}\right]
$$

with $\{(x, y) \in \Omega, \quad z>0\}$.

The velocity profile for fully developed laminar flow inside elliptical tubes is:

$$
w(x, y)=w_{0}=\text { constant }\{(x, y) \in \Omega, z>0\}
$$

In this model, the inlet condition is:

$$
T(x, y, z)=T_{0} \quad\{(x, y) \in \Omega, z>0\}
$$

The condition of constant heat flux at the boundary is given by:

$$
-k \frac{\bar{o} T(x, y, z)}{\partial \eta}=\dot{q}_{0}^{\prime \prime} \quad\{(x, y) \in \Gamma, z>0\}
$$

Finally, according to the symmetry of the problem:

$$
\begin{aligned}
& \left.\frac{\partial T(x, y, z)}{\partial x}\right|_{x=0}=0 \\
& \left.\frac{\partial T(x, y, z)}{\partial y}\right|_{y=0}=0
\end{aligned}
$$

\section{Coordinates Transformation}

The main difficulty related to the boundary conditions application to elliptical geometries has been overcome by employing the coordinate change shown in Fig. 1. 
a)

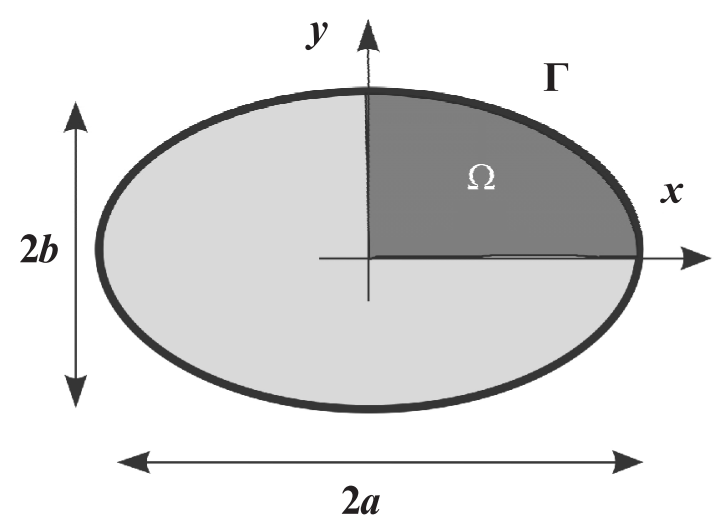

b)

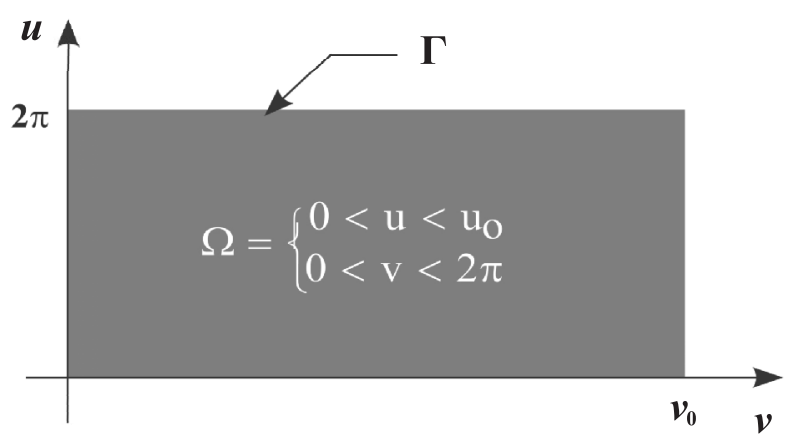

Figure 1. Geometries of the duct and coordinates.

(a) Original geometry, (b) Modified geometry.

The temperature profile and other physical parameters have been written in dimensionless form as:

$$
\begin{gathered}
\theta(X, Y, Z)=\frac{T_{0}-T(x, y, z)}{\dot{q}_{0}^{\prime \prime} D_{h} / k} \\
X=\frac{x}{D_{h}} \\
Y=\frac{y}{D_{h}} \\
Z=\frac{z}{\left(D_{h} P e\right)} \\
\eta^{*}=\frac{\eta}{D_{h}} \\
P e=\frac{\rho c w_{p} D_{h}}{k}
\end{gathered}
$$

$$
\begin{aligned}
& \alpha=\frac{a}{D_{h}} \\
& \beta=\frac{b}{D_{h}} \\
& D_{h}=\frac{4 A_{s c}}{P}
\end{aligned}
$$

$$
\rho_{\text {asp }}=\frac{b}{a}
$$

$$
A_{s c}=\pi a b
$$

$$
P=4 a \int_{0}^{\pi / 2} \sqrt{1-\kappa^{2} \sin ^{2} \theta} d \theta
$$

$$
\kappa=\frac{\sqrt{a^{2}-b^{2}}}{a}
$$

With these new variables, the energy equation becomes:

$$
\frac{\partial \theta(X, Y, Z)}{\partial Z}=\frac{\partial^{2} \theta(X, Y, Z)}{\partial X^{2}}+\frac{\partial^{2} \theta(X, Y, Z)}{\partial Y^{2}}
$$

The boundary conditions and the entrance condition are as follows:

$$
\begin{array}{ll}
\theta(X, Y, Z)=0 & \{(x, y) \in \Omega, Z=0\} \\
\frac{\bar{\partial} \theta(X, Y, Z)}{\partial \eta^{*}}=1 & \{(x, y) \in \Gamma, z>0\}
\end{array}
$$

$$
\frac{\bar{\partial} \theta(X, Y, Z)}{\partial X}=0 \quad\{X=0, Z>0\}
$$

$$
\frac{\bar{\partial} \theta(X, Y, Z)}{\partial Y}=0 \quad\{Y=0, Z>0\}
$$

The orthogonal system of elliptic coordinates $(\mathrm{u}, \mathrm{v})$ is used to transform the original domain, with elliptical contour in the coordinates $(\mathrm{X}, \mathrm{Y})$, into a domain with rectangular contour in the transformed system $(\mathrm{u}, \mathrm{v})$ : 
CIÊNCIA/SCIENCE

$$
\begin{gathered}
X=\alpha^{*} \cosh (v) \cos (u) \\
Y=\alpha^{*} \sinh (v) \sin (u) \\
Z=z \\
\alpha^{*}=\frac{\alpha}{\cosh \left(v_{0}\right)} \\
v_{0}=\operatorname{arctanh}\left(\frac{\beta}{\alpha}\right) \quad\{0<\beta<\alpha\}
\end{gathered}
$$

The coefficients $h_{u}$ and $h_{v}$ and the Jacobian $\mathrm{J}$ of the transformation of the system of coordinates $(\mathrm{X}, \mathrm{Y})$ into the system $(\mathrm{u}, \mathrm{v})$ are given by:

$$
\begin{array}{r}
h_{u}(u, v)=h_{v}(u, v)=h(u, v) \\
h(u, v)=\alpha^{*} \sqrt{\left[\sinh ^{2}(v)+\sin ^{2}(u)\right]} \\
J(u, v)=\frac{\partial(X, Y)}{\partial(u, v)}=\alpha^{* 2}\left[\sinh ^{2}(v)+\sin ^{2}(u)\right]
\end{array}
$$

With these new variables the equations of the elliptical contour and energy become, respectively:

$$
\begin{gathered}
{\left[\frac{X}{\alpha^{*} \cosh \left(v_{0}\right)}\right]^{2}+\left[\frac{Y}{\alpha^{*} \sinh \left(v_{0}\right)}\right]^{2}=1} \\
H(u, v) \frac{\partial \theta(u, v, Z)}{\partial Z}=\frac{\partial^{2} \theta(u, v, Z)}{\partial u^{2}}+\frac{\partial^{2} \theta(u, v, Z)}{\partial v^{2}}
\end{gathered}
$$

with $H(u, v)$ given by:

$$
H(u, v)=J(u, v)
$$

The entry and boundary conditions in the new coordinate system become:

$$
\begin{gathered}
\theta(u, v, Z)=0 \quad\{(u, v) \in \Omega, Z=0\} \\
\frac{\bar{o} \theta(u, v, z)}{\partial u}=0 \quad\left\{u=0,0 \leq v \leq v_{0}, Z>0\right\} \\
\frac{\bar{\partial} \theta(u, v, z)}{\partial u}=0 \quad\left\{u=\pi / 2,0 \leq v \leq v_{0}, Z>0\right\}
\end{gathered}
$$

$$
\begin{aligned}
& \frac{\dot{\partial} \theta(u, v, z)}{\partial v}=0 \quad\{0 \leq u \leq \pi / 2, v=0, Z>0\} \\
& \frac{\partial \theta(u, v, z)}{\partial v}=h(u, v)\left\{0 \leq u \leq \pi / 2, v=v_{0}, Z>0\right\}
\end{aligned}
$$

\section{Homogenization of the Boundary Conditions}

For the application of the GITT, it is convenient to implement the homogenization of the boundary conditions to increase the convergence rate of the series that represents the solution. In order to accomplish this, the following change of variables is considered:

$$
\theta(u, v, Z)=\theta^{*}(u, v, Z)+\frac{v^{2}}{2 v_{0}} h\left(u, v_{0}\right)
$$

With this new variable, the energy equation is:

$$
\begin{aligned}
& H(u, v) \frac{\partial \theta^{*}(u, v, Z)}{\partial Z}=\frac{\partial^{2} \theta^{*}(u, v, Z)}{\partial u^{2}}+ \\
& \frac{\partial^{2} \theta^{*}(u, v, Z)}{\partial v^{2}}+G(u, v)
\end{aligned}
$$

with:

$$
\begin{aligned}
G(u, v) & =\frac{h\left(u, v_{0}\right)}{v_{0}}+\frac{\alpha^{*} v^{2}}{2 h\left(u, v_{0}\right) v_{0}}\{\cos (2 u)- \\
& \left.-\left[\frac{\alpha^{*}}{2 h\left(u, v_{0}\right)} \sin ^{2}(2 u)\right]^{2}\right\}
\end{aligned}
$$

The entry and boundary conditions are then redefined by:

$$
\theta^{*}(u, v, Z)=-\frac{v^{2}}{2 v_{0}} h\left(u, v_{0}\right) \quad\{(u, v) \in \Omega, Z=0\}
$$

$$
\frac{\partial \theta^{*}(u, v, z)}{\partial u}=0 \quad\left\{u=0,0 \leq v \leq v_{0}, Z>0\right\}
$$

$\frac{\partial \theta^{*}(u, v, z)}{\partial u}=0 \quad\left\{u=\pi / 2,0 \leq v \leq v_{0}, Z>0\right\}$

$$
\frac{\partial \theta^{*}(u, v, Z)}{\partial v}=0 \quad\left\{0 \leq u \leq \pi / 2, v=v_{0}, Z>0\right\}
$$




\section{CIÊNCIA/SCIENCE}

Maia et al. Heat Transfer Study in Slug Flow on Elliptical...

\section{Application of the GITT}

To obtain the solution of the diffusion equation in the new coordinate system, Eq. (42), subjected to the conditions given by Eqs. (44)-(48), the Generalized Integral Transform Technique is applied. In order to accomplish this, the following auxiliary eigenvalue problem related to the independent variable $\mathrm{u}$ is considered:

$$
\frac{d^{2} \psi(u)}{d u^{2}}+\mu^{2} \psi(u)=0 \quad\{0 \leq u \leq \pi / 2\}
$$

with the boundary conditions:

$$
\begin{array}{ll}
\frac{d \psi(u)}{d u}=0 & \{u=0\} \\
\frac{d \psi(u)}{d u}=0 & \{u=\pi / 2\}
\end{array}
$$

The eigenvalues and eigenfunctions associated to this problem are:

$$
\begin{aligned}
& \mu_{i}=2 i \quad(i=1,2,3 \ldots) \\
& \psi_{i}(u)=\cos \left(\mu_{i} u\right)
\end{aligned}
$$

The above eigenfunctions are orthogonal, allowing the following pair inverse-transform:

$$
\begin{aligned}
& \tilde{\theta}_{i}^{*}(v, Z)=\int_{0}^{\pi / 2} K_{i}(u) \theta^{*}(u, v, Z) d u \\
& \theta^{*}(u, v, Z)=\sum_{i=1}^{\infty} K_{i}(u) \tilde{\theta}_{i}^{*}(v, Z)
\end{aligned}
$$

Where $\mathrm{K}_{\mathrm{i}}(\mathrm{u})$ are the normalized eigenfunctions given by:

$$
K_{i}(u)=\frac{\psi_{i}(u)}{N_{i}^{1 / 2}}
$$

where:

$$
N_{i}=\int_{0}^{\pi / 2} \psi_{i}^{2}(u) d u= \begin{cases}\pi / 2, & i=1 \\ \pi / 4, & i>1\end{cases}
$$

According to the formalism presented by Cotta (1998), Eq. (34) and Eq. (42) are multiplied by operators $\int_{0}^{\pi / 2} K_{i}(u) d u$ and $\int_{0}^{\pi / 2} \theta^{*}(u, v, Z) d u$, respectively. Following this procedure and applying the boundary conditions described by Eqs. (37)-(40) and Eqs. (45)-(48), the obtained system is:

$$
\begin{gathered}
\sum_{j=1}^{\infty} A_{i j}(v) \frac{\partial \tilde{\theta}_{j}^{*}(v, Z)}{\partial Z}+\mu_{i}^{2} \tilde{\theta}_{i}^{*}(v, Z)= \\
\frac{\partial^{2} \tilde{\theta}_{i}^{*}(v, Z)}{\partial u^{2}}+C_{i}(v)
\end{gathered}
$$

where:

$$
\begin{aligned}
& A_{i j}(v)=\int_{0}^{\pi / 2} K_{i}(u) K_{j}(u) H(u, v) d u \\
& C_{i}(v)=\int_{0}^{\pi / 2} K_{i}(u) G(u, v) d u
\end{aligned}
$$

Let us now consider the following eigenvalue problem related to the independent variable v:

$$
\frac{d^{2} \varphi(v)}{d v^{2}}+\lambda^{2} \varphi(v)=0 \quad\left\{0 \leq v \leq v_{0}\right\}
$$

This problem is subjected to the followings boundary conditions:

$$
\begin{array}{ll}
\frac{d \varphi(v)}{d v}=0 & \{v=0\} \\
\frac{d \varphi(v)}{d v}=0 & \left\{v=v_{0}\right\}
\end{array}
$$

The eigenvalues and the eigenfunctions for this new problem are:

$$
\begin{aligned}
& \lambda_{m}=\frac{(m-1) \pi}{v_{0}} \quad(m=1,2,3 \ldots) \\
& \varphi_{m}(v)=\cos \left(\lambda_{m} v\right) \quad(m=1,2,3 \ldots)
\end{aligned}
$$

These eigenfunctions are orthogonals and allow the following pair inverse-transform: 


$$
\begin{gathered}
\tilde{\bar{\theta}}_{i m}^{*}(Z)=\int_{0}^{v_{0}} \int_{0}^{\pi / 2} K_{i}(u) Z_{m}(v) \theta^{*}(u, v, Z) d u d v \\
\theta^{*}(u, v, Z)=\sum_{i=1}^{\infty} \sum_{m=l}^{\infty} K_{i}(u) Z_{m}(v) \tilde{\bar{\theta}}_{i m}^{*}(Z)
\end{gathered}
$$

where $Z_{\mathrm{m}}(\mathrm{v})$ are the normalized eigenfunctions:

$$
\begin{gathered}
Z_{m}(v)=\frac{\varphi_{m}(v)}{M_{m}^{I / 2}} \\
M_{m}=\int_{0}^{v_{0}} \varphi_{m}^{2}(v) d v=\left\{\begin{array}{l}
v_{0}, m>1 \\
v_{0} / 2, m>1
\end{array}\right.
\end{gathered}
$$

To determine the transformed temperature equation $\tilde{\bar{\theta}}_{i m}^{*}(Z)$, the procedure is similar to that one related to the first eigenvalue problem. Eq. (24) and Eq. (32) are multiplied by operators $\int_{0}^{v_{0}} Z_{m}(v) d v$ and $\int_{0}^{v_{0}} \tilde{\theta}^{*}(v, Z) d v$, respectively, and using boundary conditions Eqs. (37)-(40) and Eqs. (45)-(48), the following system of ordinary differential equations is obtained:

$$
\sum_{n=1}^{\infty} \sum_{j=1}^{\infty} B_{i j m n} \frac{d \tilde{\bar{\theta}}_{j n}^{*}(Z)}{d Z}+\left(u_{i}^{2}+\lambda_{m}^{2}\right) \tilde{\tilde{\theta}}_{i m}^{*}(Z)+D_{i m}=0
$$

with:

$$
\begin{aligned}
& B_{i j m n}=\int_{0}^{v_{0}} Z_{m}(v) Z_{n}(v) A_{i j}(v) d v= \\
& \int_{0}^{v_{0}} \int_{0}^{\pi / 2} K_{i}(u) K_{j}(u) Z_{m}(v) K_{n}(v) H(u, v) d u d v \\
& D_{i m}=-\int_{0}^{v_{0}} Z_{m}(v) C_{i}(v) d v= \\
& -\int_{0}^{v_{0}} \int_{0}^{\pi / 2} K_{i}(u) Z_{m}(v) G(u, v) d u d v
\end{aligned}
$$

The parameters $\mathrm{B}_{\mathrm{ijm} n}$ and $\mathrm{D}_{\mathrm{im}}$ can be integrated and, therefore, determined. The solution of this system of ordinary differential equations, when subjected to the transformed entry condition, is the following

$$
\begin{aligned}
& \tilde{\bar{\theta}}_{\mathrm{im}}^{*}(0)=\int_{0}^{\mathrm{v}_{0}} \int_{0}^{\pi / 2} \mathrm{~K}_{\mathrm{i}}(\mathrm{u}) \mathrm{Z}_{\mathrm{m}}(\mathrm{u}) \theta^{*}(\mathrm{u}, \mathrm{v}, 0) \mathrm{dudv}= \\
& =-\int_{0}^{\mathrm{v}_{0}} \int_{0}^{\pi / 2} \mathrm{~K}_{\mathrm{i}}(\mathrm{u}) \mathrm{Z}_{\mathrm{m}}(\mathrm{u}) \frac{\mathrm{v}^{2}}{2 \mathrm{v}_{0}} \mathrm{~h}\left(\mathrm{u}, \mathrm{v}_{0}\right) d u d v
\end{aligned}
$$

The transformed temperature $\overline{\tilde{\theta}}_{i m}^{*}(Z)$ is obtained from Eq. (70). Thus, the dimensionless temperature $\theta(\mathrm{u}, \mathrm{v}, \mathrm{Z})$ can be numerically evaluated using Eq. (66), just truncating the expansion in series of orthogonal functions, for a given order $\mathrm{i}=\mathrm{N}$ and $\mathrm{m}=\mathrm{M}$ :

$$
\begin{gathered}
\theta(u, v, Z)=\sum_{i=1}^{N} \sum_{m=l}^{M} K_{i}(u) Z_{m}(v) \tilde{\bar{\theta}}_{i m}^{*}(Z)+ \\
\frac{v^{2}}{2 v_{0}} h\left(u, v_{0}\right)
\end{gathered}
$$

Obviously, larger values of $\mathrm{N}$ and $\mathrm{M}$ would result in higher accuracy of the numerical results, neglecting round-off errors.

\section{Average Fluid Temperature and Nusselt Numbers}

The average fluid temperature for a given duct cross section can be determined by means of a balance of energy between the tube inlet section and the given cross section, at a position $z$ along the axis:

$$
\dot{q}_{0}^{\prime \prime} P z=\rho u_{a v} A_{s c} c_{p}\left(T_{a v}-T_{0}\right)
$$

The dimensionless average fluid temperature is defined by:

$$
\theta_{a v}(z)=4 Z
$$

The dimensionless average fluid temperature can also be determined through the integration of the temperature distribution:

$$
\theta_{a v}(Z)=\frac{D_{h}^{2}}{A_{s c}} \int_{\Omega} \theta(X, Y, Z) w(X, Y) d \Omega
$$

In the plane $(u, v), \theta_{a v}$ is expressed by the following equation: 


$$
\begin{aligned}
\theta_{a v}(Z)= & \frac{D_{h}^{2}}{A_{s c}} \int_{0}^{\pi / 2} \int_{0}^{v_{o}}\left\{\left[\sum_{i=1}^{N} \sum_{m=1}^{M} K_{i}(u) Z_{m}(v) \overline{\tilde{\theta}}_{i m}^{*}(Z)\right]\right. \\
& \left.+\frac{v}{2 v_{0}} h\left(u, v_{0}\right)\right\} H\left(u, v_{0}\right) d u d v
\end{aligned}
$$

Equations (76) and (78) are adequate for the verification of the accuracy of the numerical results when the expansion is truncated in orders $i=N$ and $m=M$.

The average wall temperature is obtained by the integration:

$$
\theta_{w, a v}(Z)=\frac{4 D_{h}}{P} \int_{0}^{\pi / 2} \sum_{i=1}^{N} \sum_{m=l}^{M} K_{i}(u) Z_{m}\left(v_{0}\right) \overline{\tilde{\theta}}_{i m}^{*}(Z) h\left(u, v_{0}\right) d u
$$

The Nusselt number is defined by:

$$
N u(z)=\frac{h(z) D_{h}}{k}
$$

with $h(z)$ defined as follows:

$$
h(z)=\frac{q_{0}^{\prime \prime}(z)}{T_{w, a v}-T_{a v}(z)}
$$

By using the dimensionless variables, the Nusselt number becomes:

$$
N u(Z)=\frac{1}{\theta_{w, a v}-\theta_{a v}}
$$

The average Nusselt number is obtained by integrating Eq. (82) along the tube axis:

$$
N u_{a v}(Z)=\frac{1}{Z} \int_{0}^{Z} N u\left(Z^{\prime}\right) d Z^{\prime}
$$

Shah and London (1978) define the thermal entry length $\left(L_{t h}\right)$ as the position where the local Nusselt number is $5 \%$ higher than the Nusselt number in the region where the fluid is thermally developed:

$$
L_{t h} \equiv \text { positive root of }\{1.05 N u(\infty)-N u(Z)=0\}
$$

\section{RESULTS AND DISCUSSION}

In order to determine the coefficients $\overline{\widetilde{\theta}}_{i m}(Z)$, the expansion given by Eq. (70) has been truncated to several choices of $\mathrm{M}$ and $\mathrm{N}$ values. Parameters $\mathrm{B}_{\mathrm{ijmn}}$ and $\mathrm{D}_{\mathrm{im}}$ have been numerically calculated by a Gauss quadrature method (36 points of quadrature) and the equation system, Eq. (74), has been solved by using the routine DIVPAG of the IMSL Fortran Numerical Library (IMSL Library, 1979).

It has been noticed that the convergence becomes slower when the aspect ratio $\mathrm{b} / \mathrm{a} \rightarrow 1$. For truncations to $\mathrm{M}$ and $\mathrm{N}$ both higher than 25 , the values of calculated Nusselt number, for $Z>0.0001$, converged within around 3 digits or more, for the analyzed cases. Therefore, all calculations in the present study truncated the expansion to $\mathrm{M}=25$ and $\mathrm{N}=25$, generating a system of 625 ordinary differential equations.

The difference between the average temperature calculated by Eq. (78) and the average temperature calculated by Eq. (76) is less than $10^{-5}$ for $Z>0.0001$. The results for average wall temperature, local and average Nusselt numbers are presented in Table 1 for aspect ratio $\mathrm{b} / \mathrm{a}=0.5$. The behavior of these parameters has been shown in Figs. 2 to 4 for several aspect ratios.

Table 1. Average wall temperature, local and average Nusselt number in the Z-axis for aspect ratio $\mathrm{b} / \mathrm{a}=0.5$

\begin{tabular}{|c|c|c|c|c|}
\hline $\mathrm{z}$ & $\theta_{\mathrm{av}}$ & $\theta_{\mathrm{w}, \mathrm{av}}$ & $\mathrm{Nu}_{\infty}$ & $\mathrm{Nu}_{\mathrm{av}}$ \\
\hline 0.0001 & 0.0004 & 0.0114 & 91.1 & 155 \\
\hline 0.0002 & 0.0008 & 0.0161 & 65.2 & 115 \\
\hline 0.0005 & 0.0020 & 0.0257 & 42.2 & 76.3 \\
\hline 0.0010 & 0.0040 & 0.0366 & 30.7 & 55.8 \\
\hline 0.0020 & 0.0080 & 0.0523 & 22.6 & 40.6 \\
\hline 0.0050 & 0.0200 & 0.0845 & 15.5 & 27.1 \\
\hline 0.0100 & 0.0400 & 0.123 & 12.1 & 20.2 \\
\hline 0.0200 & 0.0800 & 0.182 & 9.84 & 15.4 \\
\hline 0.0500 & 0.2000 & 0.321 & 8.29 & 11.4 \\
\hline 0.1000 & 0.4000 & 0.529 & 7.75 & 9.69 \\
\hline 0.2000 & 0.8000 & 0.933 & 7.52 & 8.64 \\
\hline 0.5000 & 2.0000 & 2.13 & 7.49 & 7.95 \\
\hline 1.0000 & 4.0000 & 4.13 & 7.49 & 7.72 \\
\hline 2.0000 & 8.0000 & 8.13 & 7.49 & 7.60 \\
\hline 5.0000 & 20.000 & 20.1 & 7.49 & 7.54 \\
\hline 6.0000 & 24.000 & 24.1 & 7.49 & 7.53 \\
\hline 7.0000 & 28.000 & 28.1 & 7.49 & 7.52 \\
\hline 8.0000 & 32.000 & 32.1 & 7.49 & 7.52 \\
\hline
\end{tabular}

From the obtained results it may be observed that the thermal development is slower when the aspect ratio $\mathrm{b} / \mathrm{a} \rightarrow 0$. On the other hand, it has been noticed that the values of wall temperature and Nusselt number, calculated for 
several eccentricities of ellipsis, approximate asymptotically in the region near tube entry. In the region where the flow is thermally developed a strong dependence of these parameters with the eccentricity of the ellipsis has been observed, when the aspect ratio $\mathrm{b} / \mathrm{a}<0.5$. When the aspect ratio $\mathrm{b} / \mathrm{a}>0.9$, also in the fully developed region, an asymptotical approximation of these parameters has been noticed. In particular, the asymptotical local Nusselt number is $\mathrm{Nu}=8.0$ for circular cross section in the duct.

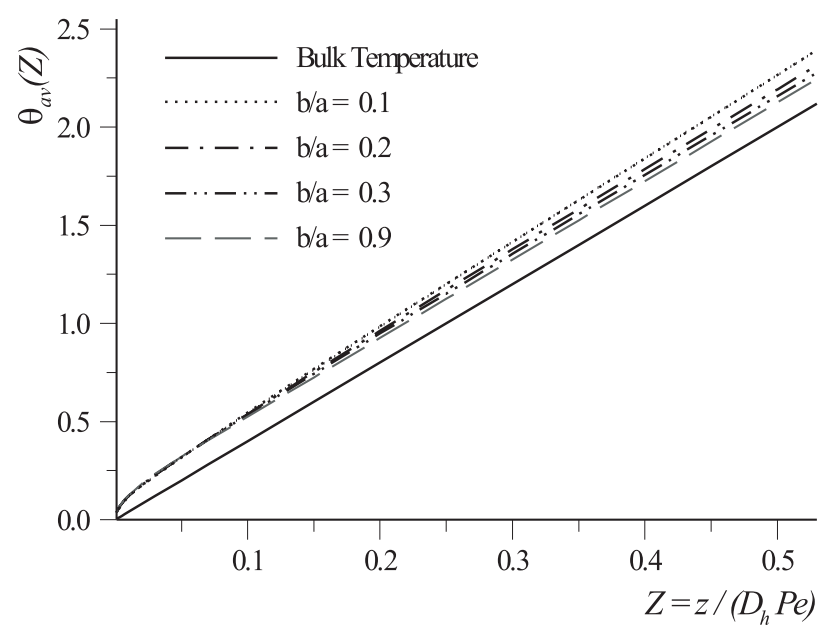

Figure 2. Fluid average temperature and average wall temperature in the Z-axis, for several aspect ratios

Thermal development occurs farther from the duct entrance when aspect ratio $\mathrm{b} / \mathrm{a} \rightarrow 0$ and at the limits of b/a 1.0 the results for the Nusselt number approximate those obtained for flows in ducts of circular section.

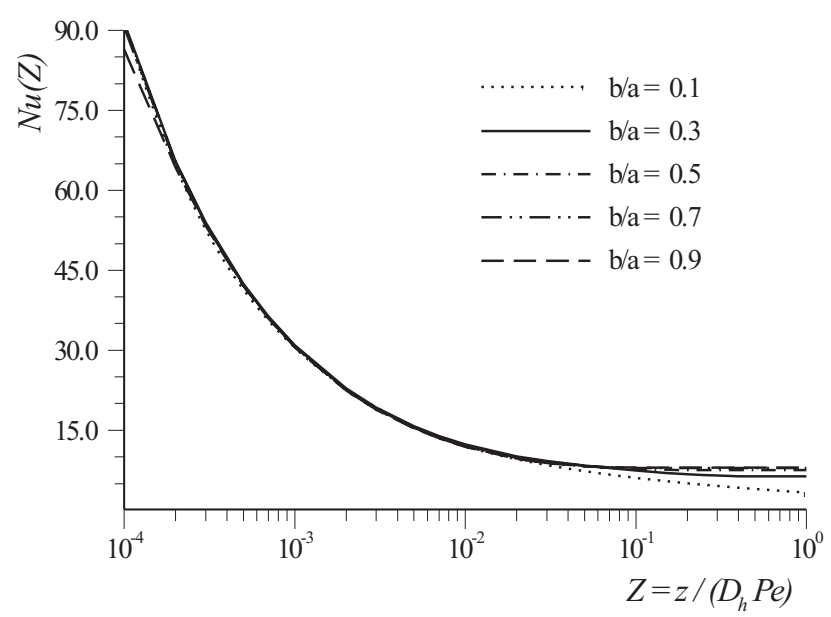

Figure 3. Local Nusselt number in the Z-axis, for several aspect ratios

Finally, Tab. 2 presents the results obtained in this work, corresponding to Nusselt numbers of thermally developed flows and Fig. 5 shows the limit Nusselt and the thermal entry length, for elliptical ducts cross section flow.

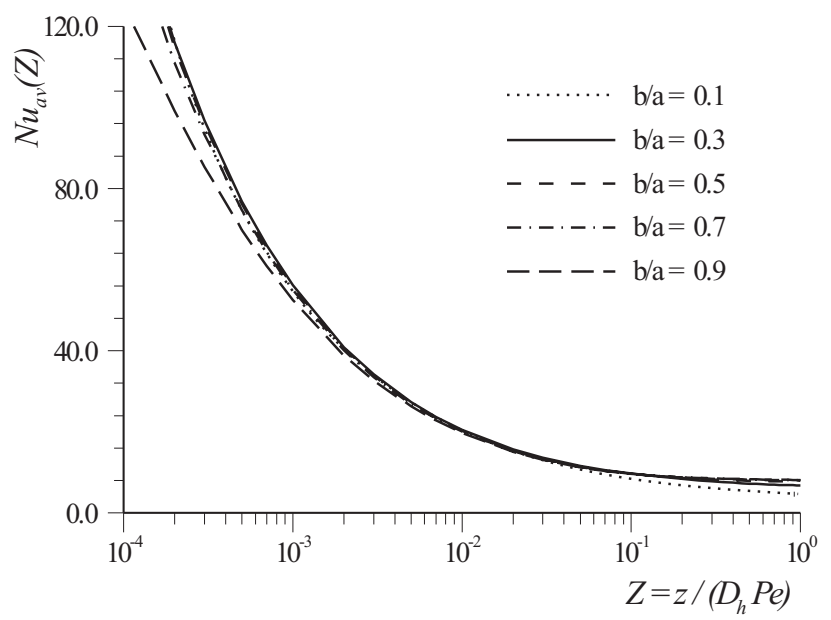

Figure 4. Average Nusselt number in the Z-axis, for several aspect ratios

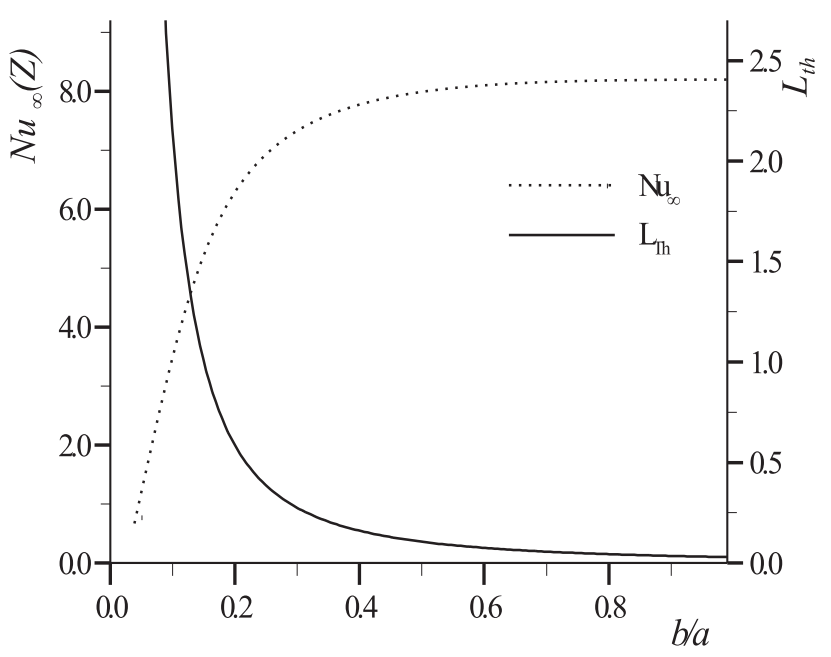

Figure 5. Limit Nusselt and the thermal entry length, for elliptical ducts cross section flow

Table 2. Nusselt number for fully developed flow and thermal entry length, for several tube aspect ratios

\begin{tabular}{|c|c|c|}
\hline $\mathrm{b} / \mathrm{a}$ & $\mathrm{Nu} \infty$ & $\mathrm{L}_{\text {th }}$ \\
\hline 0.10 & 2.293 & 2.360 \\
\hline 0.20 & 4.924 & 0.522 \\
\hline 0.30 & 6.336 & 0.228 \\
\hline 0.40 & 7.086 & 0.121 \\
\hline 0.50 & 7.469 & 0.088 \\
\hline 0.60 & 7.695 & 0.065 \\
\hline 0.70 & 7.857 & 0.050 \\
\hline 0.80 & 7.906 & 0.048 \\
\hline 0.90 & 7.929 & 0.047 \\
\hline 0.99 & 7.934 & 0.047 \\
\hline
\end{tabular}




\section{CONCLUSIONS}

In the present work, the GITT has been successfully applied to the problem of thermally developing flow inside ducts of elliptical cross section, subjected to second kind boundary conditions. The inherent difficulty of application of the boundary in problems with this geometry has been overcome by means of a change of the cartesian variables to a system of orthogonal elliptic coordinates, that transforms the contour of elliptical section of the duct to a new domain of rectangular shape. The convergence of the temperature distribution is slow and it is necessary to truncate $\mathrm{M}$ and $\mathrm{N}$ at a relatively high order. For regions near the duct entrance, $Z \approx 0.0001$, it has been necessary to truncate the series to $\mathrm{M}=\mathrm{N}>25$ to obtain a satisfactory convergence for the Nusselt number calculations, although for values of $\mathrm{Z}$ near the region of thermal development, satisfactory convergence has been obtained with fewer terms in the series. In fact, in the fully developed region, five terms in the series have shown to be usually sufficient for a good numerical convergence.

The results obtained through GITT allowed the determination of the parameters of interest, as well as the wall temperature and the local and average Nusselt number. Moreover, the results for the Nusselt number obtained for the thermally developed region exhibited excellent agreement with those reported in the literature at the limits of $\mathrm{b} / \mathrm{a} \rightarrow 1.0$ (circular section ducts).

\section{REFERENCES}

Alves, T. A., Pelegrini, M. F., Maia, C. R. M. and Ramos, R. A. V., 2004, Heat Transfer in Slug Flow Inside Elliptical Ducts, In: XXV Iberian Latin American Congress on Computational Methods (in CD-ROM), 14 p., Recife, Brazil. (in Portuguese)

Aparecido, J. B., 1997, How to Choose Eigenvalue Problems When Using Generalized Integral Transforms to Solve Thermal ConvectionDiffusion Problems, In: $14^{\text {th }}$ Brazilian Congress of Mechanical Engineering (in CD-ROM), Bauru, Brazil.

Cotta, R. M. and Özisik, M. N., 1986, Laminar Forced Convection in Ducts with Periodic Variation of Inlet Temperature, Journal of Heat and Mass Transfer, Vol. 29, No. 10, pp. 1495-1501.
Cotta, R. M. and Ozisik, M. N., 1987, Diffusion Problems With General Time-Dependent Coefficients, Brazilian Journal of Mechanical Sciences, Vol. 9, No. 4, pp. 269-292.

Cotta, R. M., 1998, The Integral Transform Method in Thermal and Fluids Science and Engineering, Begell House Inc., New York.

Diniz, A. J., Aparecido, J. B. and Cotta, R. M., 1990, Heat Conduction with Ablation in a Finite Slab, Heat and Technology, Vol. 8, pp. 30-43.

IMSL Library, 1979, Edition 7, GNB Building, 7500 Ballaire Blod, Houston, Texas 77036.

Kakaç, S., Shah, R. K. and Aung, W., 1998, Handbook of Single-Phase Convective Heat Transfer, John Wiley, New York.

Maia, C. R. M., Aparecido, J. B. and Milanez, L. F., 2000, Heat Transfer in Laminar Forced Convection Inside Elliptical Tube with Boundary Condition of First Kind, In: $3^{\text {rd }}$ European Thermal Sciences, Heidelberg, Germany, Vol. 1.

Maia, C. R. M., Aparecido, J. B. and Milanez, L. F., 2004, Thermally Developing Forced Convection of Non-Newtonian Fluids Inside Elliptical Ducts, Heat Transfer Engineering, Vol. 25, No. 7, pp. 13-22.

Pelegrini, M. F., Alves, T. A., Maia, C. R. M. and Ramos, R. A. V., 2004, Heat Transfer in Slug Flow in Elliptical Ducts, In: $10^{\text {th }}$ Brazilian Congress of Thermal Engineering and Sciences (in CD-ROM), 15 p., Rio de Janeiro, Brazil.

Shah, R. K. and London, A. L., 1978, Laminar Flow Forced Convection in Ducts, Advances in Heat Transfer, Supplement 1, Academic Press Inc., New York, 477 p.

Silva, J. B. C. and Cotta, R. M., 1992, Solutions Simultaneously Developing Laminar Flow Inside Parallel Plate Channels, International Journal of Heat and Mass Transfer, Vol. 35, No. 4, pp. 887-895.

Vick, B. and Wells, R. G., 1986, Laminar Flow with an Axially Varying Heat Transfer Coefficient, International Journal of Heat and Mass Transfer, Vol. 29, pp. 1881-1889. 